\title{
A cost-effective decentralized vehicle remote positioning and tracking system using BeiDou Navigation Satellite System and Mobile Network
}

\author{
Jingfa Wei ${ }^{1}$, Chui-Hui Chiu'², Feijiang Huang ${ }^{3,5,6}$, Junjun Zhang ${ }^{3}$ and Chenglin Cai ${ }^{4,5,6^{*}}$
}

\begin{abstract}
Most existing production vehicle tracking services rely on the Global Positioning System (GPS) and a proprietary server/client infrastructure. This type of inflexible and centralized architecture incurs severe vender-dependency and high service cost. In this paper, we propose a cost-effective decentralized vehicle remote positioning and tracking system architecture. This architecture only consists of two components: (1) a vehicle terminal for collecting real-time vehicle location from a navigation satellite system and report it to a user terminal via the existing mobile network and (2) a user terminal on a mobile phone for analyzing the location information from (1) and inform the owner. We prototype the positioning and tracking system using the BeiDou Navigation Satellite System (BDS) and Global System for Mobile Communication(GSM) Network. Experiment results illustrate that the system is capable of tracking vehicle location and warning the owner if the vehicle unexpectedly moves more than $100 \mathrm{~m}$ at low cost. With maximum vender-neutrality and high-availability as the design philosophy, the system is able to utilize multiple available navigation satellite systems and mobile networks.
\end{abstract}

Keywords: Vehicle monitoring, Vehicle positioning and tracking, BDS, GSM mobile network, Application (APP) monitoring

\section{Introduction}

With the improvement of people's living standards, the number of private cars is increasing. But after owners parking their vehicles in parking lots, they might have difficulties in finding their vehicles, or their vehicles might be stolen. In the early days, more attention was paid to vehicle anti-theft, so we designed mechanical, chip-styled and other vehicle anti-theft systems [1,2]. Electronic and network vehicle monitoring systems have emerged along with the development of electronic technology, network technology, satellite navigation technology, etc. How to quickly determine the location of vehicles after parking or after losing vehicles has become the main research

\footnotetext{
* Correspondence: csdxgxgl@sina.com

${ }^{4}$ School of Information Engineering, Xiangtan University, Xiangtan 411105, China

${ }^{5}$ School of Information and Communication, Guilin University of Electronic Technology, Guilin 541004, China

Full list of author information is available at the end of the article
}

direction of vehicle monitoring [3-6]. Yongwei and others introduced the GSM network into the car alarm system for remote monitoring. When the vehicle is stolen, it can send an alarm signal in a short message and cutoff the automobile circuit and oil circuit [7]. Tianzi studied the remote transmission of automobile position when the automobile ignition switch was triggered illegally. The user can be informed of the car's situation and its real-time latitude and longitude data through the liquid crystal display (LCD) monitor in the monitoring center to take an emergent action [8]. In view of vehicle safety problems such as vehicle theft and unauthorized driving, Xiaoli used GPS module to collect real-time information such as latitude and longitude, altitude, vehicle speed, and other information, achieving real-time vehicle tracking and positioning, vehicle trajectory reporting, and anti-theft [9]. Jian proposed an intelligent monitoring system for logistics vehicles consisting of three parts: GPS vehicle terminal, monitoring center server group, and control terminal [10]. 
To sum up, most of the existing vehicle monitoring systems use GPS to collect vehicle location information, and domestic autonomy is week. The location information is sent by using existing mobile communication network. We have set up corresponding database servers and monitoring centers to store the vehicles' conditions and remotely controlled vehicles by the monitoring center. In the monitoring center, we can only check the vehicles' functioning conditions. To develop such a system is very complex and costly, and its maintenance requires a lot of manpower and money. Individuals need to register as members and pay fees before they use the system. Therefore, the cost of using such system is high for individual owners $[11,12]$. With the gradual improvement of BeiDou Navigation Satellite System, the vehicle monitoring system using BeiDou Navigation Satellite System has also become a research hotspot [13-15]. In this paper, a vehicle monitoring and locating tracking system based on BDS/GSM is designed and implemented. The BeiDou Navigation Satellite System developed by China spots the vehicle location. The location information of the vehicle is sent through the GSM network. The system monitors the APP client by itself. It can check the vehicles' positions on mobile phones at any time and monitor the vehicle in real time. When there is no need to monitor the vehicle, this system can be used to find vehicles in parking lots.

\section{Key technology of vehicle remote positioning}

\subsection{The composition and positioning principle of the} BeiDou Navigation Satellite System

BDS is a global satellite navigation system developed by China. In the constellations of BDS, to achieve continuous global coverage and regional enhancement, three orbital satellites have been designed: the Geosynchronous Earth Orbit satellite (GEO satellite), the Inclined Geo Synchronous Orbit satellite (IGSO satellite), and the Medium Earth Orbit satellite (MEO satellite). The system space constellation consists of five GEO satellites and 30 non-geostationary orbit (Non-GEO) satellites. The GEO satellites were located at $58.75^{\circ}$ (GEO01 satellite), $80^{\circ}$ (GEO02 satellite), $110.5^{\circ}$ (GEO03 satellite), $140^{\circ}$ (GEO04 satellite), and $160^{\circ}$ (GEO05 satellite), respectively. The non-GEO satellite is composed of $27 \mathrm{MEO}$ satellites and three IGSO satellites, of which the orbit height of the MEO satellite is $21,500 \mathrm{~m}$, the orbit angle is $55^{\circ}$, and it is evenly distributed on the three orbital surfaces. The IGSO satellite orbit is $36,000 \mathrm{~km}$ high, evenly distributed on the three inclined track surfaces. The orbit angle is $55^{\circ}$, and the three IGSO satellites' sub-satellite point trajectory coincides. The intersection points are $118^{\circ}$ east, and the phase difference is $120^{\circ}[16,17]$.

The basic principle of BDS is to take the instantaneous position of the satellite as the known starting data, and to determine the location of the points measured by space distance resection method. As shown in Fig. 1, if we assume the coordinate of the point to be measured as $(x, y, z)$, the clock difference of the installed BeiDou satellite-positioning receiver is $b$. By measuring the time $\Delta t$ required to send the navigation signals from the BeiDou satellite to the receiver, and the satellite ephemeris received by the BeiDou positioning receiver from which more than four satellites' coordinates $\left(x_{i}, y_{i}, z_{i}, i=1,2,3,4\right)$ can be gained, we can have the following four equations [17].

$$
\begin{aligned}
& \sqrt{\left(x_{1}-x\right)^{2}+\left(y_{1}-y\right)^{2}+\left(z_{1}-z\right)^{2}}+c b=\rho_{1} \\
& \sqrt{\left(x_{2}-x\right)^{2}+\left(y_{2}-y\right)^{2}+\left(z_{2}-z\right)^{2}}+c b=\rho_{2} \\
& \sqrt{\left(x_{3}-x\right)^{2}+\left(y_{3}-y\right)^{2}+\left(z_{3}-z\right)^{2}}+c b=\rho_{3} \\
& \sqrt{\left(x_{4}-x\right)^{2}+\left(y_{4}-y\right)^{2}+\left(z_{4}-z\right)^{2}}+c b=\rho_{4}
\end{aligned}
$$

The unknown coordinates of the measured point $x, y, z$ and the clock difference of the receiver $b$ can be obtained

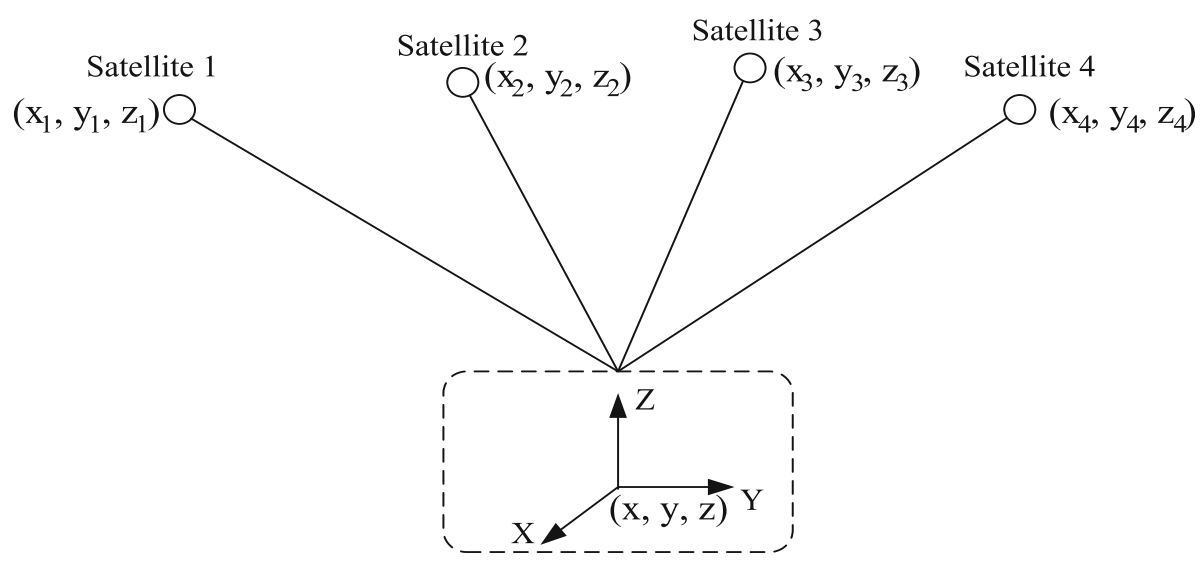

Fig. 1 Schematic diagram of BDS positioning calculation 
through the above four equations. $\rho_{i}=c \cdot \Delta t_{i}(i=1,2,3,4)$ is the pseudorange between the satellite and the receiver; $c$ is the speed of light.

\subsection{GSM technology}

GSM, a digital mobile communication standard developed by the European Telecommunications Standard Institute (ETSI), has been adopted by more than 100 countries worldwide. GSM standard equipment occupies more than $80 \%$ of the current global cellular mobile communication equipment market. It is currently the most widely used mobile phone standard. China has established a GSM mobile communication network covering the whole country. Short message service (SMS) is a value-added service of the GSM system. It uses the signaling channel to transmit information, and its transmission mode is to be stored and forwarded first, that is, after the short message is sent out, it will be stored in the short message center (SMC) first and then forwarded by SMC to the receiver. SMS are transmitted through a wireless control channel, which can be stored and forwarded through SMC. Each short message is limited to $140 \mathrm{~B}$ (140 characters or 70 Chinese characters).

The short message center has the function of short message storage, and the message will not be lost when the terminal equipment is turned off. By using the bidirectional transmission characteristics of short messages, remote control and information collection of all kinds of electrical equipment can be realized, that is, remote control and telemetry. This research uses GSM short message technology to realize the remote transmission of vehicle location information. It has the advantages of wide coverage, small investment, and less operating cost and has unparalleled advantages which other means of communication does not possess [18].

\subsection{Single-chip computer control technology}

Single chip is a perfect microcomputer system, which integrates the functions of a central processor, random memory, a variety of $\mathrm{I} / \mathrm{O}$ ports and interrupts, and timers to a piece of silicon by using the technology of ultra-large scale integrated circuit. Therefore, it is called a single-chip microcomputer and is a kind of programmable operation. Because of its unique manufacturing technology and hardware structure design, the single chip microcomputer has the advantages of fast running speed, strong control ability, low power consumption, low voltage, easy to produce, and portable carrying. It has good expansibility and strong practical application [19]. In this study, we use microcontroller unit (MCU) to filter the vehicle longitude and latitude information through program operation and initially monitor the movement state of the vehicle. On this basis, the geographic information is processed and the geographic information is transformed into text form by the serial port communication module of the single-chip computer, and then the GSM module sends messages to owners' mobiles.

\subsection{Android system}

Android system, an open-source software, was originally created by Andy Rubin, which was later acquired by Google. The open-source mobile operating system, developed by Google and the open mobile alliance, is mainly used for mobile devices. It is the most influential mobile terminal operating system in the world. The bottom of the Android system is built on top of the Linux system. The system consists of four layers: application software, user interface, middleware, and operation system. Its construction uses a layer of software layer, which separates layer from layer and defines the division of labor in each layer. This division also ensures low coupling between layers and layers. When the lower layer or internal layer changes, the upper application does not have to do any change [20]. Based on the Android operating system, this study will realize real-time display of vehicle location and voice alerts after vehicle moving on mobile phones.

\section{System requirement analysis and overall plan design}

3.1 System basic demand analysis

By summarizing the advantages and disadvantages of various existing vehicle monitoring systems, according to the actual situation of the current vehicle monitoring system, we conclude that this system is mainly concerned with the use of individual owners and should have the following basic functions. It can collect the information of vehicle position in real time. It can also process data of vehicle location and make judgments on the change of vehicle location according to the situation of vehicle movement. The vehicle location information can be transmitted to the owner's personal terminal for long distance. The personal terminal can capture the vehicle location information in real time and display the vehicle location information on the terminal. At the same time, the vehicle's movement can be monitored by voice prompt.

\subsection{System overall plan design}

According to the above basic requirement analysis, the design of the remote vehicle monitoring system based on individual user is mainly composed of three parts: vehicle terminal, user terminal, and mobile network. The terminal of the vehicle is mainly composed of three parts: main control module, BDS module and GSM module. The user terminal mainly consists of the owner's phone and the monitoring APP. The communication between vehicle terminal and user terminal is realized through the existing 
mobile communication network, to achieve remote transmission of vehicle location information, as shown in Fig. 2. The main control module of the vehicle terminal is to locate the vehicle latitude and longitude information, conduct speed judgment, and control message sending of the vehicle location. The BDS module mainly collects the vehicle location information. The GSM module mainly sends short messages of vehicle location. The user terminal is mainly to capture the short message of the vehicle location, display the vehicle position on the terminal's electronic map, and give voice alarm to the owner.

\section{Implementation of vehicle terminal}

\subsection{Main hardware components of the vehicle terminal}

The hardware of the vehicle terminal mainly consists of the main control chip STM32F103C8T6, BDS module UM220-III, GSM module SIM900A, reset module, and power module, as shown in Fig. 3. The reset module can reset the microcontroller manually when the single-chip program does work abnormally so that it can work normally. The power module is connected to the system through a Universal Serial Bus (USB) to DC5.0 interface source, which is convenient for testing and mobile. The mobile power supply is used. The BDS module is responsible for receiving BeiDou satellite navigation signals and calculating vehicle location information. The GSM module is responsible for sending vehicle location messages through the mobile terminal network to the user terminal.

In the vehicle remote positioning system, the selection of the main control chip will affect the cost and processing capability of the whole system. After the previous study, the STM32F103C8T6 chip, a widely used single-chip computer, produced by the Italian group is used. The kernel of STM32F103C8T6 is based on ARM32 bit Cortex-M3 CPU, which has a 32-bit CPU on a single-chip and system programmable flash.
The BDS module is UM220-IIIN, which is the BDS/ GPS dual system module designed by the core star technology limited company. It is mainly used for vehicle monitoring and navigation, handheld equipment, and so on. UM220-IIIN adopts the low power Global Navigation Satellite System(GNSS) SoC chip-HumbirdTM whose intellectual property is owned by the company. It is the smallest and fully domestic BDS/GPS module in the market. It has the characteristics of high integration and low power consumption. It has a strong advantage in the BeiDou application that has strict requirements on size and power consumption.

The GSM module uses the SIM900A module produced by SIMCom Company and uses the SMT (Surface Mount Technology) package dual frequency GSM/GPRS (General Packet Radio Service) module solution. The kernel uses the ARM9216EJ-S kernel, which can meet the requirements of cost and size. GSM is mainly connected to the single-chip computer through a serial port, so as to realize the control of the GSM module by a single-chip microcomputer. The connection to the single chip is the standard serial port, which supports AT (Attention) instruction and $2 \mathrm{G}$ (second-generation cellular technology) internet access.

\subsection{Vehicle terminal software implementation}

The software realization of the vehicle terminal mainly consists of the main program, the BDS data receiving and processing program, the GSM SMS sending program, and the analog serial port program. The programming language uses the $C$ language. The main program flow is shown in Fig. 4. The main program is to initialize each module first, and the BDS module automatically initializes the hardware after switching on the power supply. After each module is initialized, the parking location is sent to the owner by message so that the owner

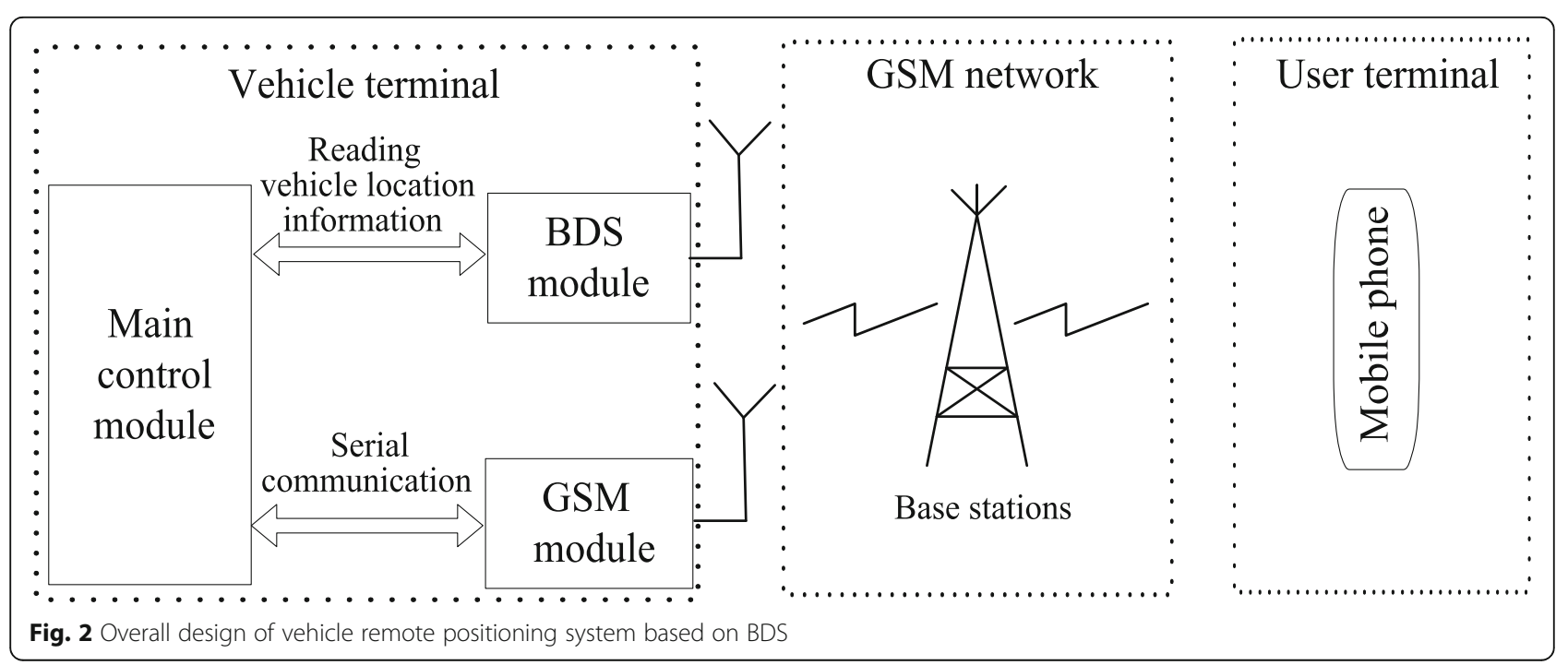




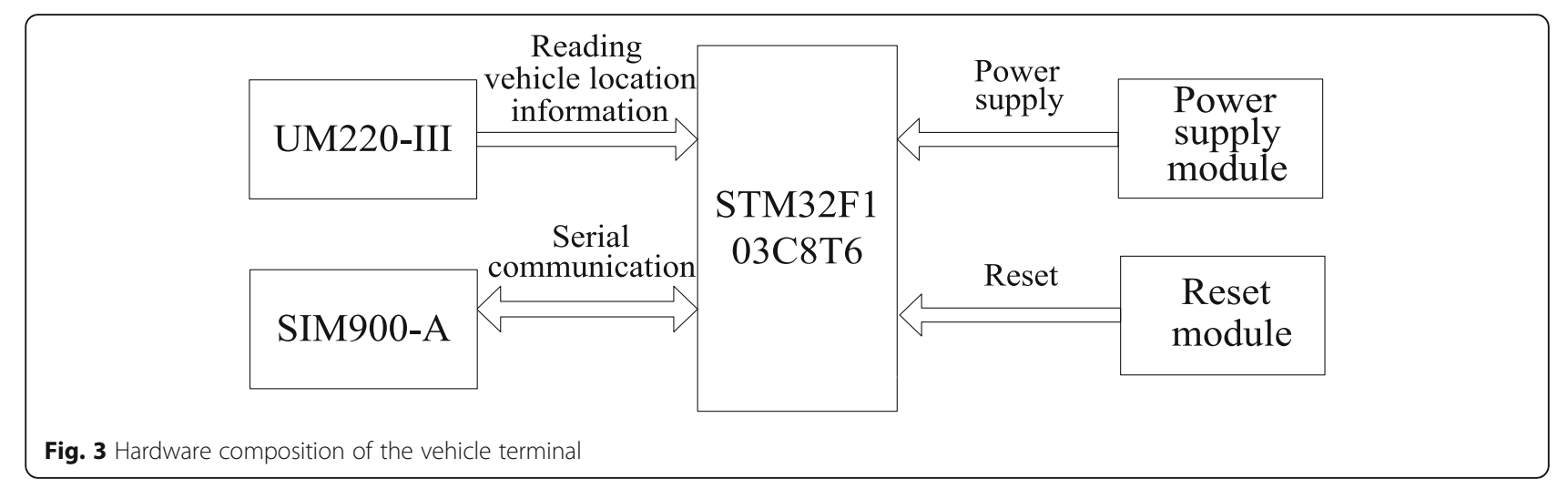

can see the location of its own car on the electronic map. Then it goes into the cycle, collects the vehicle location data by BDS module every other time, and sees if the latitude and longitude are changed. It is specific to judge whether the distance of the vehicle's latitude and longitude exceeds the specified threshold, and the distance $d$ of latitude and longitude on the earth is calculated according to the following formula (5).

The longitude and latitude of point $\mathrm{A}$ in the formula are $\lambda_{A}$ and $\phi_{A}$, respectively. The longitude and latitude of point $\mathrm{B}$ are $\lambda_{B}$ and $\phi_{B}$, respectively. If the distance of the vehicle's current latitude and longitude exceeds the

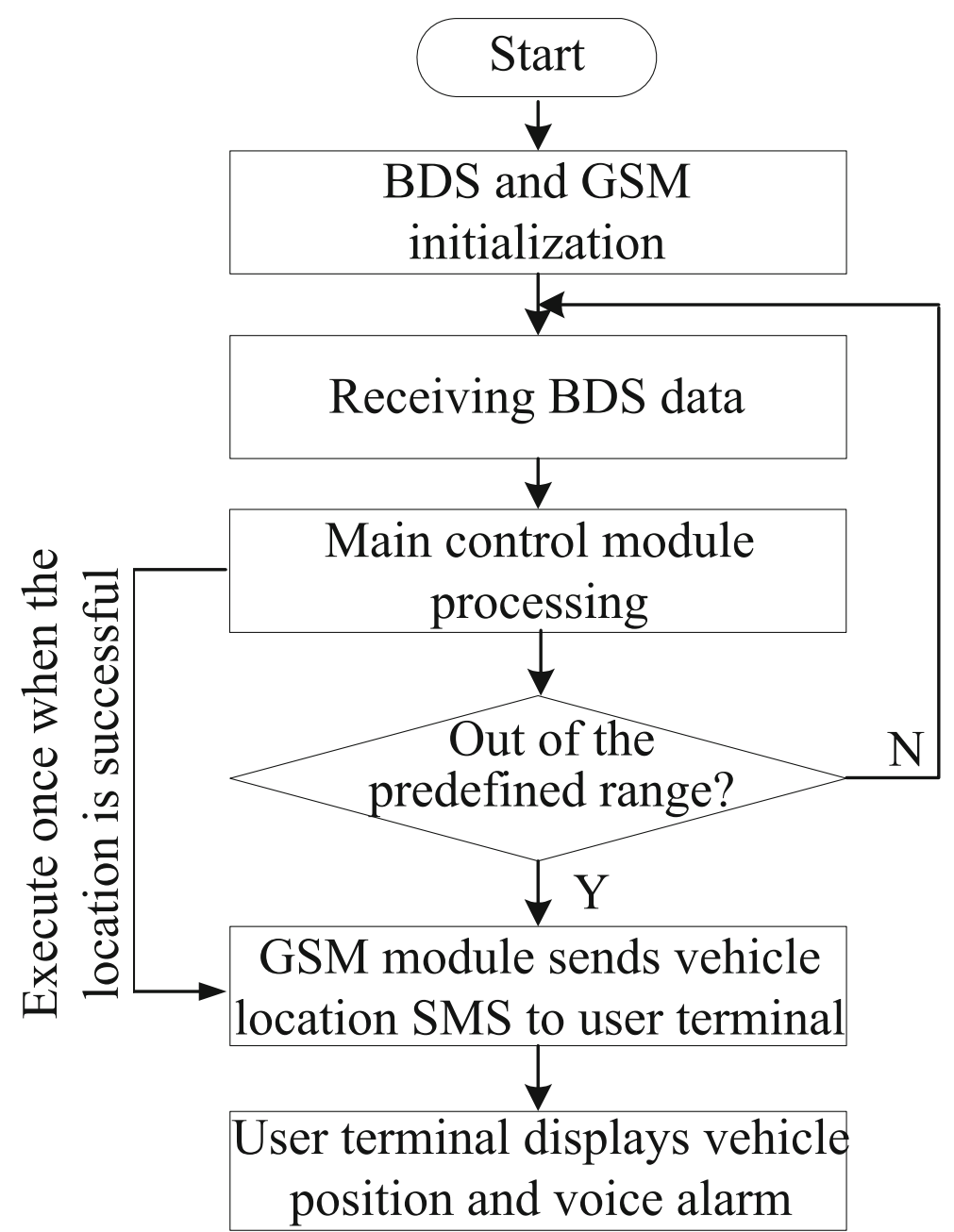

Fig. 4 Main program flow chart 
specified range threshold and has speed, the vehicle is judged as unintentional movement, and the vehicle terminal sends the vehicle location message to the user terminal.

$$
d=111.12 \cos \left[\frac{1}{\sin \phi_{A} \sin \phi_{B}+\cos \phi_{A} \cos \phi_{B} \cos \left(\lambda_{B}-\lambda_{A}\right)}\right]
$$

When the BDS module is in working, the BDS satellite positioning information will be transmitted through the related serial port to the master chip (the single chip). After receiving the data transmitted from BDS, the MCU first judges the frame head of the data frame, extracts the frame of the "\$GPGLL" field, and gets the location information through processing. The receiving and processing flow of the BDS positioning data is shown in Fig. 5. The longitude and latitude of the single-chip computer reads out from the \$GPGLL data format are $d d m m$. mmmmmm format that is not converted, that is, the degree format, which needs to be converted to the $d d$. $d d d d d d$ format first, and the calculation method is as follows.

$$
d d . d d d d d=d d+m m . m m m m m m / 60
$$

The communication between the GSM module and the main chip is realized through a serial port, and the program execution flow is shown in Fig. 6. The data transmission of GSM is usually implemented by the AT instruction set. When the MCU implements these instructions, it must be the ASCII code of the characters. The beginning of AT instruction generally has a specific command prefix, and the end is a command end identifier. The command prefix is usually made up of two characters in AT, and the end flag of the command is generally a return key. After each AT instruction is sent out, a result is returned to answer for the received command. When setting message for GSM, we use "AT" online instruction. "AT $+C P M S=|S M|$ " sets the short message storage location; "AT+CMGF $=1$ " instruction

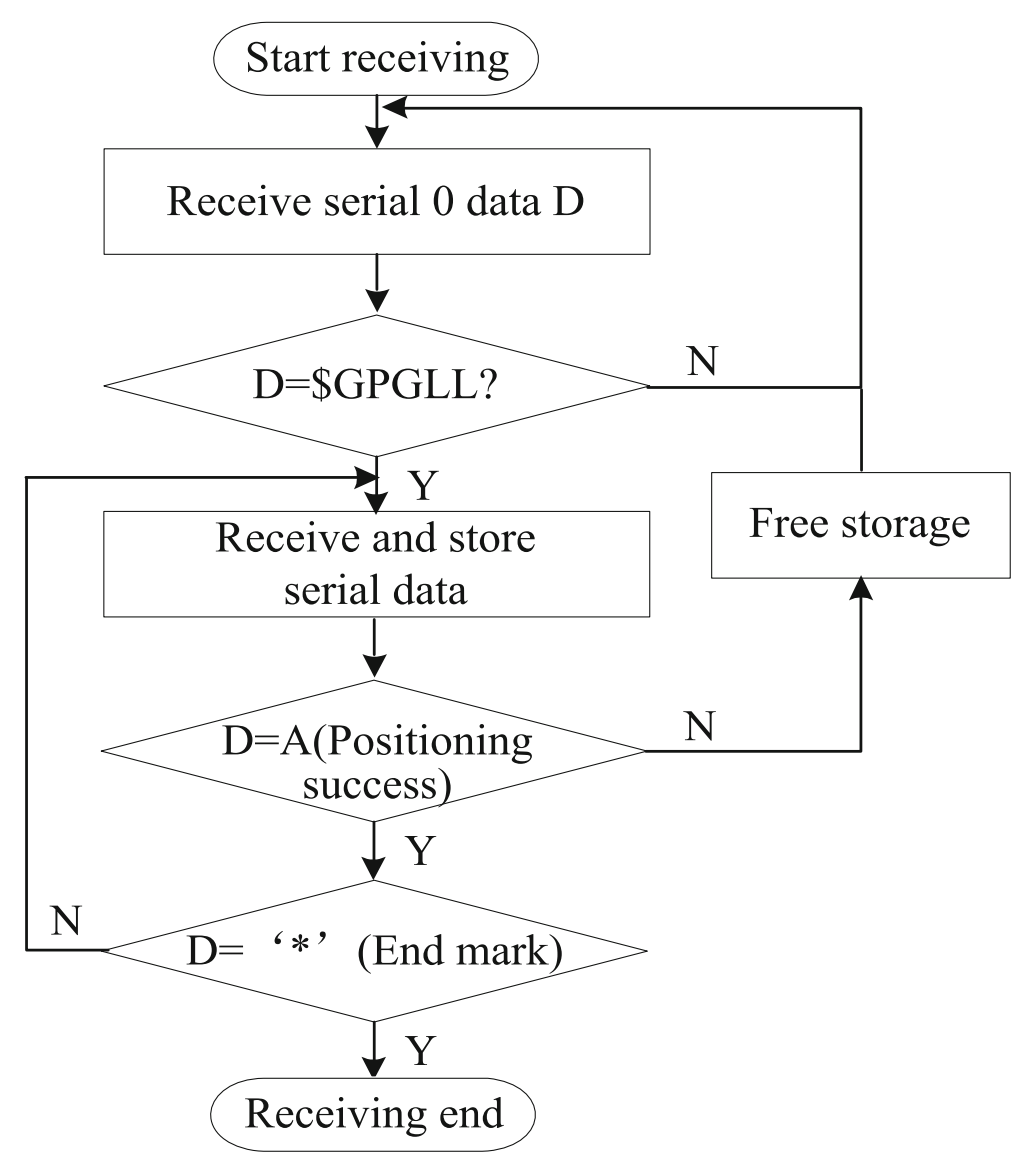

Fig. 5 BDS data receiving and processing program flow chart 


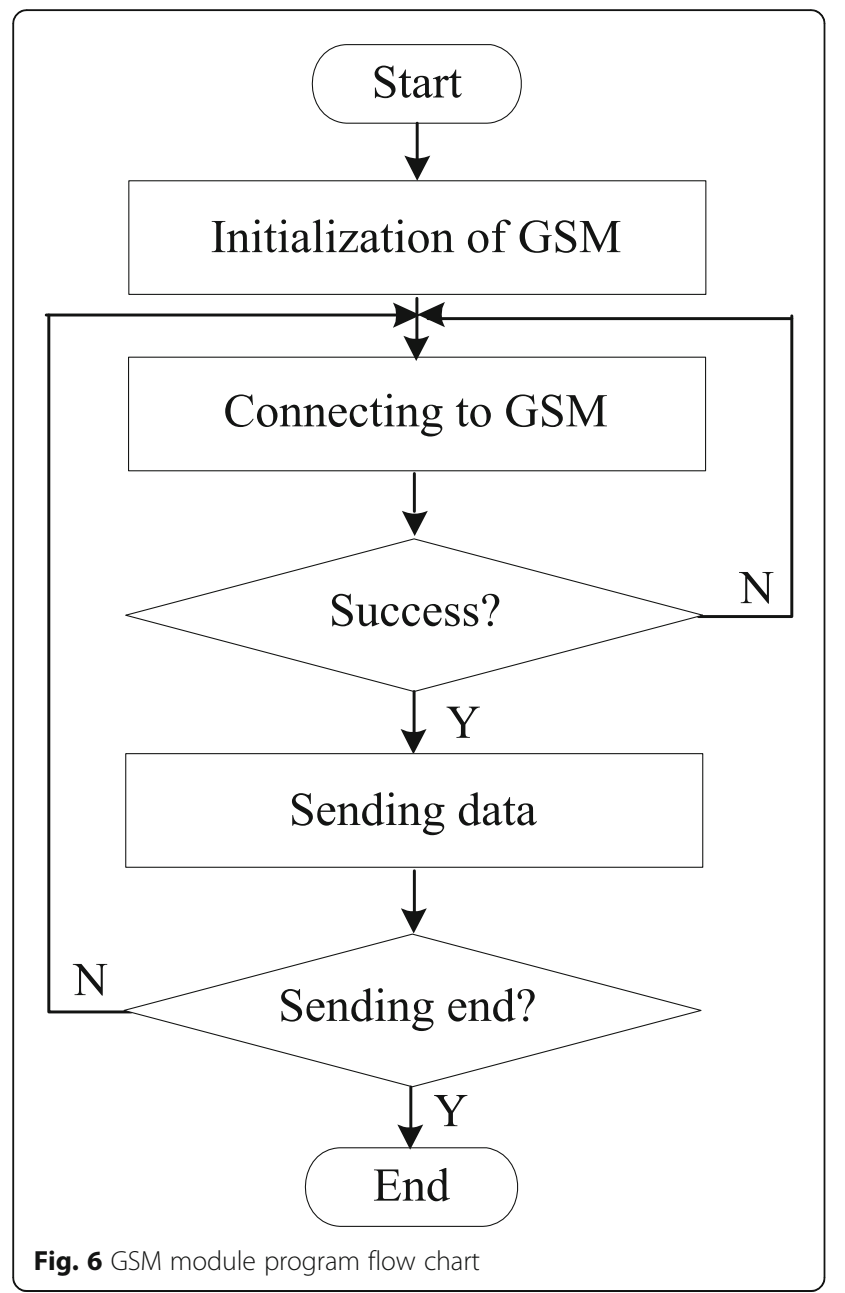

sets the text message to text; "AT+CMGS=" instruction sets the destination phone number and message content.

The program of the MCU control module is divided into an analog serial port subroutine and serial port interrupt service program. The process of serial port interrupt service program execution is shown in Fig. 7. The analog serial port subroutine is 1 or 0 for the two input and output pins of the STM32F103C8T6 microcontroller. Usually, 0 stands for low level and 1 for high level. The serial port interrupt service program is mainly to send data to the GSM module, and the serial port needs to set the interrupt in the execution of the interrupt mode. When the data is sent, the ES is set 0 to close the serial interrupt and then the data to be sent to the SBUF register. When the serial port sends only data that is not accepted, the initialization process only needs to explain the work way.

\section{Realization of user terminal and system test results analysis}

The realization of the user terminal is mainly realized by the APP installed on the owner's mobile phone. It mainly

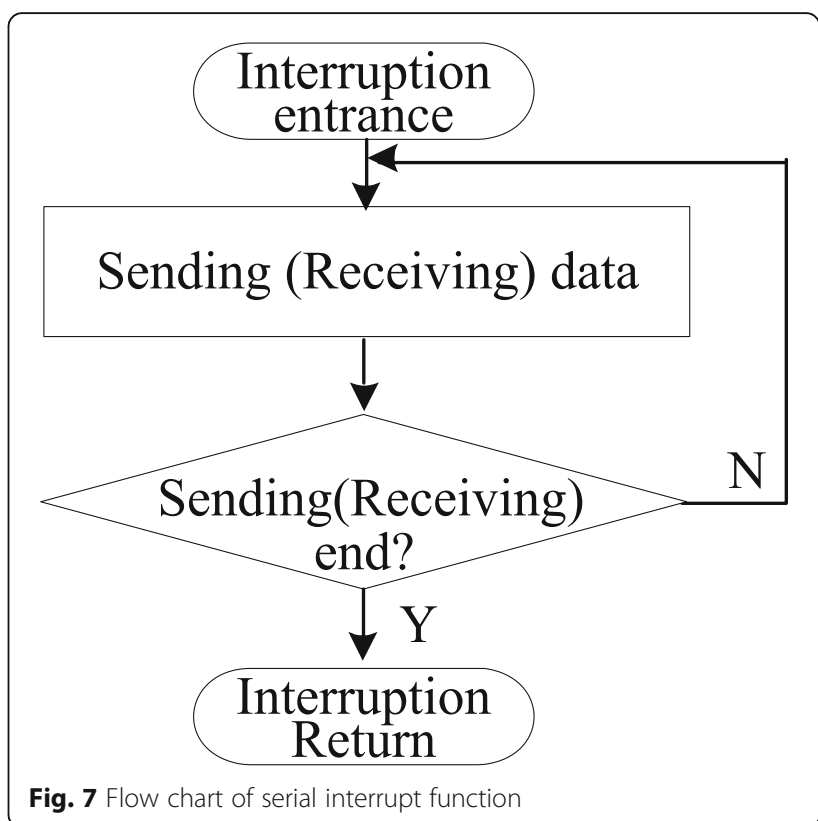

includes the functional modules such as SMS monitoring, vehicle location display, and voice play. The monitor module is used to determine whether to receive the message of the vehicle location information. The display module is used to display the extracted vehicle location information in real time on the electronic map. The voice playback module is used to simultaneously broadcast the voice alarm information of the vehicles that are previously recorded, which indicates that the vehicle owner has left the original parking position. The program flow is shown in Fig. 8.

\subsection{SMS monitoring and processing}

The method of getting SMS about the vehicle location on the mobile terminal is to set up a short message broadcast monitor on the Android terminal. When a new message is received, the monitoring system can monitor the content of the SMS latitude/longitude that has been formatted on the hardware, parsed it, and extracted the latitude and longitude in the content by using the word character capture. After that, it is passed to the activity in the display map.

\subsection{System test methods and results analysis}

The vehicle terminal with the SIM card is placed in the test car, and the SIM card number of the vehicle terminal is inputted on the user mobile phone monitoring APP. When leaving the test vehicle, the vehicle terminal power is turned on, and the user's mobile phone APP will display the current vehicle parking location, as shown in Fig. 9. This location can also be used to provide the user with the ability to find a parking location for a car in a large parking lot, when the vehicle is not 


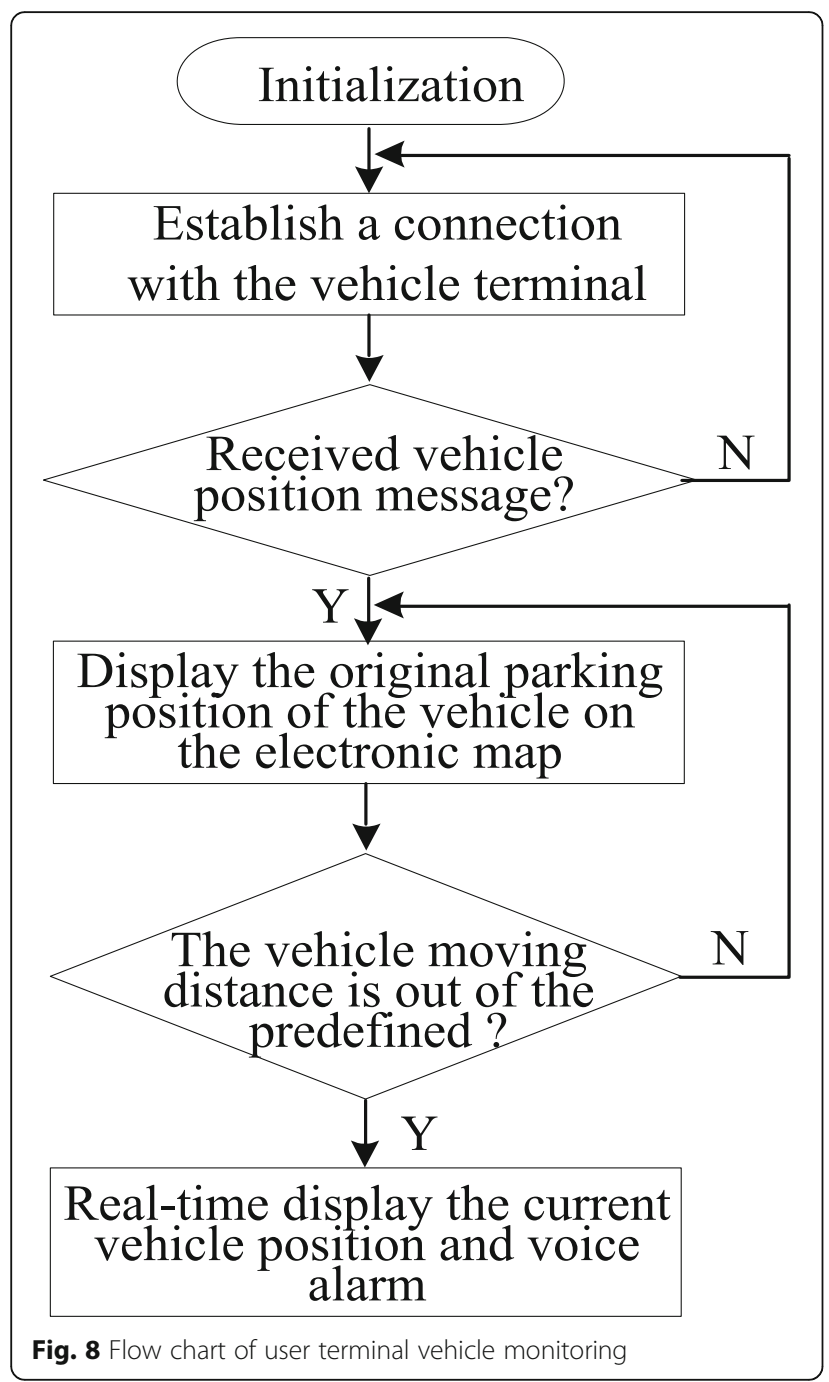

moving abnormally. The parked test vehicle is driven away from the original parking location in four different directions. When the vehicle leaves the set threshold range of $100 \mathrm{~m}$, the monitoring APP will display the new location of the vehicle again, trigger the voice alarm mechanism, and play the corresponding voice alarm to inform the car owner that the car has left the original parking location. When the vehicle continuously moves, the system will continuously update the vehicle location according to the set time interval, and the positioning tracking of the vehicle is realized.

\section{Conclusions}

In view of the continuous improvement of the BeiDou Navigation Satellite System, the coverage of the satellite navigation system has developed from the regional system to the world. Combing with the monitoring demand of the individual car owners, this paper designed and realized a remote monitoring system based on the combination of BDS and GSM. After testing, we found out when the vehicle left the original parking location 100 $\mathrm{m}$, the system could display the vehicle's location in real time, then sent out the voice alarm information at the owner's mobile phone, and realized the remote positioning and tracking of the vehicle. The system does not need back-end database support, and users do not have to pay registration fees. It only requires low price hardware costs and mobile network SMS fees. Based on daily use of mobile phones, it realizes using mobile phones to monitor the vehicle. After further optimization, it can also be widely used for tracking persons or items. This remote positioning and tracking system architecture can also be realized by other satellite navigation systems and mobile networks and has wide application prospects.

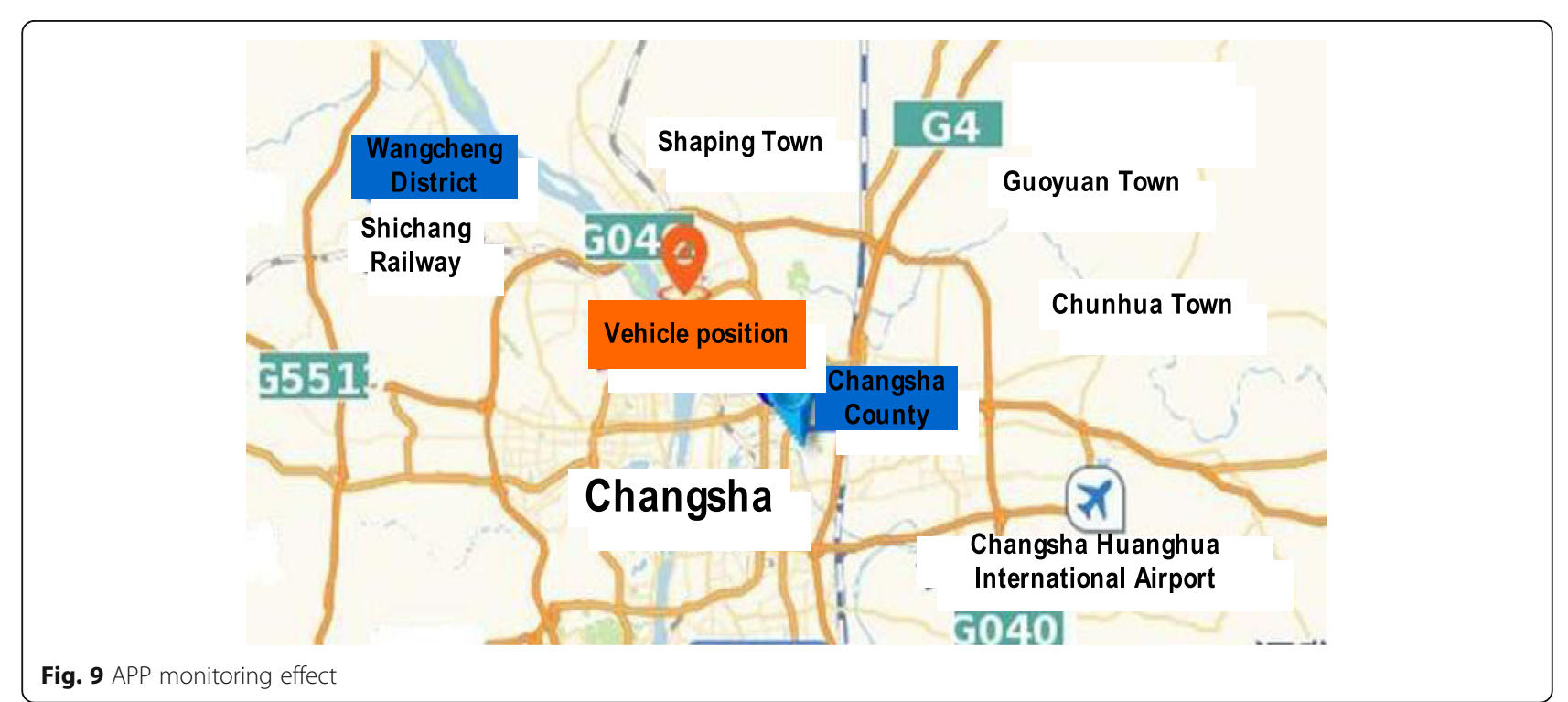




\section{Abbreviations}

APP: Application; BDS: BeiDou Navigation Satellite System; CPU: Central processing unit; GEO: Geosynchronous Earth Orbit; GNSS: Global navigation satellite system; GPRS: General Packet Radio Service; GPS: Global Positioning System; GSM: Global System for Mobile Communication; IGSO: Inclined Geo Synchronous Orbit; MEO: Medium Earth Orbit; SMC: Short message center; SMS: Short message service

\section{Acknowledgements}

The authors would like to thank all kinds of funds for funding.

\section{Funding}

This study was supported by the National Natural Science Foundation of China (no.61771150), Hunan Provincial Natural Science Foundation of China (no.2015JJ2016), Projects of Guangxi Key Research(no. AB17129028), Guangxi Colleges and Universities Key Laboratory of Satellite Navigation, and Position Sensing, Youth Talent Support Plan and Scientific Research Foundation of Changsha University (no.SF1615).

\section{Availability of data and materials}

Data sharing not applicable to this article as no datasets were generated or analyzed during the current study.

\section{Authors' contributions}

All authors have contributed to this research work. All authors have read and approved the final manuscript.

\section{Competing interests}

The authors declare that they have no competing interests.

\section{Publisher's Note}

Springer Nature remains neutral with regard to jurisdictional claims in published maps and institutional affiliations.

\section{Author details}

${ }^{1}$ The 20th Research Institute of China Electronic Technology Group Corporation, Xi'an 710068, China. ${ }^{2}$ Division of Computer Science and Engineering, Louisiana State University, Baton Rouge, LA 70803, USA. ${ }^{3}$ School of Electronic Information and Electrical Engineering, Changsha University, Changsha 410022, China. ${ }^{4}$ School of Information Engineering, Xiangtan University, Xiangtan 411105, China. ${ }^{5}$ School of Information and Communication, Guilin University of Electronic Technology, Guilin 541004, China. ${ }^{6}$ Guangxi Colleges and Universities Key Laboratory of Satellite Navigation and Position Sensing, Guilin 541004, China.

Received: 10 December 2018 Accepted: 18 April 2019

Published online: 03 May 2019

\section{References}

1. S. Zhengnan, J. Haobin, M. Shidian, Research of the vehicle anti-theft system based on fingerprint identification technology. Automob. Parts 11, 20-22 (2014)

2. Z. Mingjun, K. Lingchao, H. Chuanyi, Present status and quality analysis fo the mechanical automobile anti-theft device products in China. Automob. Parts 1, 73-75 (2014)

3. Y. Haiyang, C. Zhiliang, L. Shaobo, Design of car alarm system based on radio frequency identification technology. Comput. Meas. Control 24(2) 144-146 (2016)

4. C. Lina, Design of car security system based on GSM and GPS. Agric. Equip. Veh. Eng. 53(1), 14-18 (2015)

5. L. Xu, H. Ye, P. Tao, Research and implementation of vehicle real-time monitoring system based on android. Comput. Sci. Appl. 7(2), 109-116 (2017)

6. W. Simin, X. Yangyang, Z. Ling, L. Yaling, Intelligent anti-lost vehicle and vehicle positioning system. Electron. World 19, 37 (2014)

7. J. Yongwei, H. Ren, S. Zhu, Design and implementation of vehicle remote monitoring intrusion alarm system based on GSM network. Softw. Guid. 15(4), 92-95 (2016)

8. J. Tianzi, Design and implementation of vehicle GPS Monitoring System Based on GSM Communication Telecom World, vol 6 (2016), p. 91

9. R. Xiaoli, Vehicle positioning and monitoring system based on GPS. Comput. Meas. Control 24(2), 74-76 (2016)
10. G. Jian, Design of intelligent monitoring system for logistics vehicle based on GPS. Comput. Meas. Control 25(9), 87-90,109 (2017)

11. P. Yuanwu, Analysis and improvement of GPS vehicle monitoring system. China ClO News 7, 117-118 (2015)

12. W. Hao, L. Yan, W. Beiping, Design and implementation of GPS vehicle monitoring center system based on sky map. Geospat. Inf. 1, 42-44 (2015), 47

13. Y. Yuanxi, X. Yangyin, L. Jinlong, Y. Cheng, Progress and performance evaluation of BeiDou global navigation satellite system: Data analysis based on BDS-3 demonstration system. Sci. China Earth Sci. 48(5), 584-594 (2018)

14. W. Shuqin, W. Shenghe, Research on high performance condition monitoring system for large scale operation vehicles. J. Xi'an Univ. (Nat. Sci. Ed.) 21(2), 67-71 (2018)

15. S. Qiming, L. Xu, W. Junpeng, Civilian market situation and development prospect of Beidou satellite. J. Beijing Univ. Posts Telecommun. (Soc. Sci. Ed. ) 20(1), 95-104 (2018)

16. W. Ershen, Y. Xiaodong, H. He, Q. Pingping, Simulation and global coverage analysis of Beidou navigation satellite system. Telecommun. Eng. 56(8), 919$922(2016)$

17. F. Yongtao, L. Cairong, Preliminary analysis and discussion of the measurement precision of BeiDou satellite navigation system. Space Electron. Technol. 12(3), 55-58 (2015)

18. L. Hongchao, W. Yuanming, H. Xiaoyu, L. Jing, X. Benxian, Design of 10 kV line switch state signal acquisition device based on GSM short message technology. Instrum. Technol. 6, 33-37 (2018)

19. G. Jie, Application of single chip microcomputer in mine electric automation. Electron. Test 16, 108-188 (2017)

20. L. Jiangping, Z. Lei, The research of mobile phone video surveillance based on Andriod. J. Inner Mongolia Agric. Univ. (Nat. Sci. Ed.) 38(6), 68-72 (2017)

\section{Submit your manuscript to a SpringerOpen ${ }^{\circ}$ journal and benefit from:}

- Convenient online submission

- Rigorous peer review

- Open access: articles freely available online

High visibility within the field

- Retaining the copyright to your article

Submit your next manuscript at $\boldsymbol{\nabla}$ springeropen.com 